CARNETS DE Carnets de géographes

GÉOGRAPHES.

$14 \mid 2020$

Varia

\title{
Faire de la recherche en Chine
}

Contraintes et enseignements du terrain en contexte étranger

Basile Michel et Annie Ouellet

\section{(2) OpenEdition}

Journals

Édition électronique

URL : https://journals.openedition.org/cdg/5277

DOI : $10.4000 /$ cdg. 5277

ISSN : 2107-7266

Éditeur

UMR 245 - CESSMA

Référence électronique

Basile Michel et Annie Ouellet, "Faire de la recherche en Chine », Carnets de géographes [En ligne], 14 I

2020, mis en ligne le 20 décembre 2020, consulté le 22 mai 2021. URL : http://

journals.openedition.org/cdg/5277; DOI : https://doi.org/10.4000/cdg.5277

Ce document a été généré automatiquement le 22 mai 2021.

\section{(c)}

La revue Carnets de géographes est mise à disposition selon les termes de la Licence Creative

Commons Attribution - Pas d'Utilisation Commerciale - Pas de Modification 4.0 International. 


\title{
Faire de la recherche en Chine
}

\author{
Contraintes et enseignements du terrain en contexte étranger
}

Basile Michel et Annie Ouellet

\section{Introduction}

1 Le terrain occupe une place déterminante dans la recherche en géographie (Claval, 2013). Pourtant, les écrits portant un regard réflexif sur les enjeux et les contraintes auxquels est confronté le géographe ${ }^{1}$ lors de sa pratique du terrain sont apparus tardivement dans l'histoire de la discipline ${ }^{2}$. Entendu selon le double-sens d'un « espace comme objet de la recherche » et d'un "espace comme support de la méthode " (Calbérac, $2010: 350$ ), chaque terrain possède ses propres contraintes, que ce soit en termes d'accès aux acteurs, de délimitation, de disponibilité des données, etc., forçant le géographe à s'adapter, voire parfois à bricoler (Lemercier et al., 2013). Les terrains étrangers où le chercheur ne maîtrise pas pleinement la culture, la langue et les codes locaux constituent l'une de ces situations contraignantes amenant le géographe à s'interroger sur son positionnement, sur ses méthodes et ses outils, voire sur sa manière de penser et de comprendre les rapports entre les sociétés et l'espace (Sanjuan, 2008).

2 Cet article mobilise deux recherches menées en Chine : l'une sur la patrimonialisation et la mise en tourisme de quartiers urbains chinois, l'autre sur les dynamiques urbaines, artistiques et touristiques des quartiers créatifs de villes chinoises (Encadré 1). Ces recherches font suite à des travaux réalisés sur des thématiques similaires en contexte français (Michel, 2017, 2018; Ouellet, 2015, 2017). Intégrée dans un projet scientifique consacré à la géographie du tourisme en Chine ${ }^{3}$, la poursuite de ces travaux dans le contexte chinois a nécessité le développement d'une appréhension singulière du terrain pour faire face aux contraintes qui se sont présentées au cours de la recherche. Dans les deux cas, la non-maîtrise du mandarin a constitué la principale contrainte, bien que d'autres soient également apparues. Notre objectif est de rendre compte à la fois des obstacles qui se présentent au chercheur sur un terrain dont il ne maitrise pas la langue d'usage, et les arrangements, les hybridations et les bricolages 
qui peuvent être mis en place pour les surmonter et permettre à la recherche de progresser malgré tout. Cet objectif apparaît d'autant plus déterminant dans le contexte actuel de la recherche qui voit grandir le fonctionnement par projets et les contrats à court terme (Roddaz, 2017).

3 Cet article est structuré en deux parties. La première expose les contraintes initiales communes aux deux recherches menées en Chine. Deux types de contournements méthodologiques sont ensuite abordés : l'observation puis l'entretien directif et l'usage de l'anglais.

Encadré 1. Thématiques et terrains de recherche

Cet article est fondé sur deux recherches menées séparément par deux chercheure-s. Notre appartenance commune à l'Institut conjoint des universités de Ningbo et d'Angers à Ningbo a permis l'émergence d'une collaboration scientifique portant sur les aspects méthodologiques des enquêtes de terrain en Chine.

La première recherche porte sur l'articulation entre patrimonialisation et mise en tourisme dans le cas d'un des quartiers « historiques » de la ville de Ningbo :

NanTangLaoJie (Nantang Old Street). Ses habitants ont été délogés et une partie des bâtiments a été démolie et reconstruit selon un style " ancien ». Le quartier a été inauguré en 2012 avec une volonté affichée de mise en tourisme. Il regroupe de nombreux commerces en lien avec la gastronomie (restaurants, vente à emporter...), un hôtel, des librairies, des boutiques d'artisanat et de souvenirs, etc.

La seconde recherche porte sur les clusters créatifs ancrés dans des quartiers urbains au travers du cas du M50 à Shanghai. Cet ancien quartier industriel a été spontanément investi par des artistes et des galeristes dès les années 1980, avant d'être officiellement labellisé par les autorités locales en 2005 et de devenir l'un des « clusters créatifs » les plus reconnus du pays. Il est aujourd'hui le lieu d'une forte agglomération d'activités culturelles et créatives et attire un nombre important de visiteurs nationaux et internationaux.

\section{Les contraintes initiales inhérentes au contexte chinois : explicitation des conditions de recherche}

4 Cette première partie s'articule en trois temps. Elle présente d'abord les contraintes initiales liées à nos projets de recherche, avant d'exposer les spécificités du contexte chinois qui peuvent avoir des effets sur le travail de terrain mené, puis de conclure sur l'enjeu de la transposition de notions forgées en Occident vers la Chine.

\section{Contraintes et limites initiales}

5 Les deux recherches menées s'inscrivent dans un contexte de départ comparable en termes de méthodologie, de positionnement vis-à-vis du terrain chinois et de moyens.

6 Premièrement, dans la continuité de nos recherches précédentes, la méthodologie qualitative était privilégiée avec la volonté initiale d'utiliser l'entretien semi-directif comme outil d'enquête principal. Deuxièmement, il s'agissait dans les deux cas d'une 
première expérience de recherche en contexte non européen et nous ne maîtrisions pas la langue locale, le mandarin. L'absence de maîtrise de la langue de la population enquêtée se justifie par le contexte de la recherche. Il s'agit dans les deux cas de contrats courts, qui incluent des missions d'enseignement en plus de la recherche, ne permettant qu'un séjour de recherche de quelques mois et rendant impossible l'apprentissage du mandarin au-delà de quelques bases.

7 Troisièmement, les moyens financiers et humains alloués au projet dans lequel s'inscrivent nos recherches se sont avérés moins importants que prévu. Tout d'abord, peu de collaborations avec les enseignants-chercheurs chinois ont pu être développées car ces derniers font face à de lourdes et diverses charges de travail qui limitent leur implication dans des projets annexes ${ }^{4}$. Cette réalité nous a amené à développer nos recherches dans un contexte d'isolement partiel, avec peu de relais pour entrer sur le terrain chinois. Ensuite, des contraintes financières imprévues ont empêché le recours à des interprètes qui devaient initialement nous accompagner sur le terrain, accentuant les contraintes inhérentes à toutes recherches en terrain étranger. Afin de pallier à ces contraintes inattendues, nous avons pu nous appuyer sur l'aide de quelques étudiantes chinoises inscrites dans le cursus sino-français de l'Institut conjoint à Ningbo. Leur travail s'est déroulé dans un cadre pédagogique de formation par la recherche et n'était donc pas rémunéré. Le fait de travailler avec des étudiantes, et non des interprètes professionnels, a limité les attentes que nous pouvions avoir quant aux traductions des entretiens. De plus, la non rémunération de leur travail nous a amené à limiter leur investissement dans nos recherches, en particulier dans le cas de Shanghai au vu de l'éloignement géographique. Toutefois, cette situation offre un cadre propice à l'enrichissement mutuel: les chercheur-e-s bénéficient des compétences linguistiques des étudiantes tandis que ces dernières profitent des savoir-faire méthodologiques des premiers.

8 Ces différentes contraintes font partie des difficultés classiques auxquelles se confrontent les chercheurs en terrain étranger, avec des contextes parfois encore plus contraignants (zone rurale isolée, zone de guerre...). Dans notre cas, ces contraintes nous ont amené dès le début de la recherche au constat suivant: il est difficile, voire impossible, d'utiliser l'entretien semi-directif comme nous le souhaitions initialement.

\section{Contexte politique}

9 Au-delà de ces contraintes initiales liées à notre non maîtrise de la langue et aux conditions matérielles et humaines du projet de recherche, le contexte politique spécifique de la Chine est également porteur d'enjeux pour la réalisation d'un travail d'enquêtes sur le terrain. Malgré son ouverture économique grandissante à l'international, le pays reste encore largement marqué par un système politique autoritaire dominé par le Parti communiste chinois (PCC) (Cabestan, 2014). L'information, notamment d'ordre politique, demeure contrôlée par les autorités chinoises afin de préserver l'image positive du PCC. Cette réalité se retranscrit tout particulièrement dans le domaine de l'art où les créations d'artistes sont surveillées et font face au risque de la censure si elles ne respectent pas l'idéologie du PCC (Keane, 2016 ; Zheng et Chan 2014).

10 En conséquence, les discours des acteurs interrogés qui peuvent être recueillis en entretien, en particulier ceux des artistes et des galeristes dans le cas du M50 à 
Shanghai, doivent être considérés avec précaution, la liberté de parole étant potentiellement limitée. Il s'agit également pour le chercheur de veiller à ne pas mettre ses interlocuteurs dans l'embarras, voire en danger, en cherchant à tout prix à obtenir des informations sur des sujets sensibles tels que la politique dans le contexte chinois. Dans un souci d'éthique, cette situation entraine pour le chercheur une partielle autocensure (ESO (collectif), 2008).

\section{L'enjeu de la transposition de notions occidentales en Chine}

11 En complément, nos thématiques de recherche invitent à s'interroger sur la transposition de notions forgées en Europe et en Amérique du Nord dans le contexte chinois.

Premièrement, en partant $\mathrm{du}$ constat que le patrimoine est un construit social (Lazzarotti, 2003) dont la conception varie d'une société à l'autre, la question de la transférabilité de cette notion apparait particulièrement stimulante dans le contexte de mondialisation favorisant les échanges. En Chine, cette notion existe depuis la dynastie des Tang (618-907) et les termes employés pour la désigner ont, comme en français, connu des évolutions constantes, de yichan (" biens hérités des ancêtres »), à lishi guji (renvoyant à l'idée de monuments historiques) jusqu'à wenhua yichan (patrimoine culturel) apparu en 1985 lors de l'adhésion du pays à la Convention du patrimoine mondial portée par l'Unesco (Zhang, 2017). Le sens attribué au patrimoine diffère largement entre l'Europe et la Chine. En Europe, malgré un élargissement progressif du champ patrimonial, l'ancienneté et l'authenticité sont encore les deux critères principaux permettant d'établir la valeur du patrimoine (Coëffé et Morice, 2017). En Chine, sa valeur tient davantage à ce qu'il représente et à la mémoire qui lui est associée (Zhang, 2017).

Deuxièmement, l'analyse dans le contexte chinois des clusters créatifs, initialement développés en Europe et en Amérique du Nord, nécessite la prise en compte de la force de la circulation des modèles urbains à l'échelle mondiale, tout en considérant les spécificités liées au contexte local. En effet, à partir de 2005 et sous l'appellation chuangyi jiju, de nombreux clusters créatifs ont été créés en Chine en suivant le modèle occidental qui a séduit les élites politiques du pays par sa capacité à concilier les logiques économiques capitalistes et les principes de coopération et de collectivisme promus par le PCC (Keane, 2011). Cette réplication des clusters créatifs européens et nord-américains s'accompagne toutefois d'ajustements au contexte politique local. La surveillance des artistes et autres acteurs du monde de l'art mentionnée précédemment se matérialise concrètement dans les clusters créatifs. Leur gestion est assurée par des organisations placées directement sous le contrôle des autorités afin de rendre compte des créations qui y sont développées (Zheng et Chan 2014).

En définitive, que ce soit dans le cas du patrimoine ou des clusters créatifs, la transposition de notions occidentales dans le contexte chinois impose au chercheur de déconstruire les cadres théoriques préétablis avant d'entrer sur le terrain afin d'être en mesure de saisir pleinement les singularités locales. Par ailleurs, ce travail de transposition et de déconstruction nourrit la recherche en renseignant la circulation dans le monde des modèles (comme les clusters créatifs) et des notions (comme le patrimoine) et leurs ajustements suivant les spécificités locales. 


\section{Contournements méthodologiques. « Faire avec » les contraintes}

15 Face aux contraintes initiales de la recherche et au contexte spécifique de la Chine, il a été nécessaire de réaliser des ajustements dans notre approche du terrain. Cette deuxième partie détaille donc les deux principaux contournements méthodologiques mis en place pour pallier la difficulté à réaliser des entretiens semi-directifs.

\section{L'observation comme premier contournement}

16 Pour contourner le problème de la langue et entrer malgré tout au contact du terrain, une première adaptation méthodologique a été mise en place via la réalisation d'un travail d'observation. Considérée comme une «technique contraignante de recueil de matériau et une pratique réflexive conduisant à sa mise en ordre analytique » (Arborio, $2007: 27$ ), l'observation directe permet d'analyser précisément les pratiques et les interactions sociales dans l'espace en s'affranchissant des discours construits et contrôlés des enquêtés (Arborio et Fournier, 2015), ce qui se révèle d'autant plus précieux au vu du contexte de contrôle et de censure de l'information en Chine.

Grâce aux phases d'observation à NanTangLaoJie et au M50, il a été possible de saisir des premiers éléments de compréhension de ces deux territoires en rapport avec nos questionnements initiaux. Dans les deux cas, l'observation portait principalement sur l'organisation spatiale du quartier (degré d'enclavement, répartition des différents types d'activités), les usages et les usagers des lieux (importance de la fréquentation, pratiques et interactions...). Des séances d'observation ont été conduites à différents moments de la journée, en semaine et week-end, durant et hors période de vacances, permettant de cerner les évolutions de l'investissement de ces lieux. Ces séances d'observations régulières et planifiées ont été complétées par de l'observation " exploratoire» (Morange et al., 2016:65) dans d'autres quartiers créatifs et d'autres quartiers « anciens » respectivement à Shanghai et à Ningbo. En tant que chercheur-e-s étranger-ère-s, ces différentes observations nous ont permis d'entrer sur le terrain sans y appliquer un cadre d'analyse préconçu. Nous avons ainsi pu nous laisser surprendre par les différences et les similarités existant entre nos terrains chinois et ceux étudiés précédemment en contexte européen. Toutefois, certaines limites sont apparues.

18 Si l'observation « relève d'une géographie sensorielle » (Ibid.: 64), du fait de la barrière de la langue, la dimension visuelle a occupé une place centrale dans le cadre de nos enquêtes. Malgré cette prédominance de la vue, d'autres sens ont été sollicités. Entre autres, la dimension sonore a été mobilisée afin de distinguer, parmi les usagers des lieux, les chinois des étrangers ${ }^{5}$. En effet, sans pouvoir mener une conversation en mandarin, nous étions en mesure de reconnaître cette langue lors des phases d'observation et donc d'affiner notre connaissance des usagers des quartiers ${ }^{6}$. Si le fait de ne pouvoir comprendre qu'en partie ce qui était dit sur le terrain par les personnes observées a pu générer un sentiment de frustration, cela a permis de développer une forme de compensation sensorielle en incitant à être davantage attentif aux modes d'expression orale des individus observés.

19 Par ailleurs, la simple présence sur place du chercheur induit une perturbation de l'environnement observé (Ibid.). Dans une démarche réflexive, il s'agit donc de 
questionner le degré de perturbation provoqué par l'observateur. Notre statut de chercheur-e étranger-ère a pu constituer un élément biaisant l'observation (Vivet, Ginisty, 2008). Le degré de perturbation a néanmoins été plus important dans le cas de NanTangLaoJie qu'au M50. Dans le premier cas, la présence d'étrangers est très faible. Le quartier attire essentiellement des résidents ou des touristes nationaux (Figure 1). Ainsi, la présence d'une Occidentale munie d'un carnet de notes suscitait fréquemment des réactions, entrainnant une partielle modification de l'environnement observé. À maintes reprises, des visiteurs remarquaient cette présence étrangère, se retournant sur leur passage, s'arrêtant pour regarder le contenu du carnet de notes alors que des enfants pointaient du doigt la chercheure tout en la désignant oralement comme Měiguó Ren (Américaine). Ces réactions peuvent traduire un biais induit par la présence de la chercheure. Mais elles confortent aussi certaines informations relatives à notre terrain (cette fois au sens d'espace comme objet d'étude) : NanTangLaojie est un lieu essentiellement pratiqué par des résidents de Ningbo ou des touristes nationaux, la présence d'étrangers demeurant anecdotique. Dans le second cas, la présence d'étrangers est habituelle (Figure 2). Le M50 est aujourd'hui reconnu internationalement comme un quartier artistique ouvert au public, attirant de ce fait des visiteurs d'horizons divers. Dès lors, un chercheur européen peut facilement réaliser des observations incognito.

Figure 1. Terrain enquêté : NanTangLaoJie à Ningbo

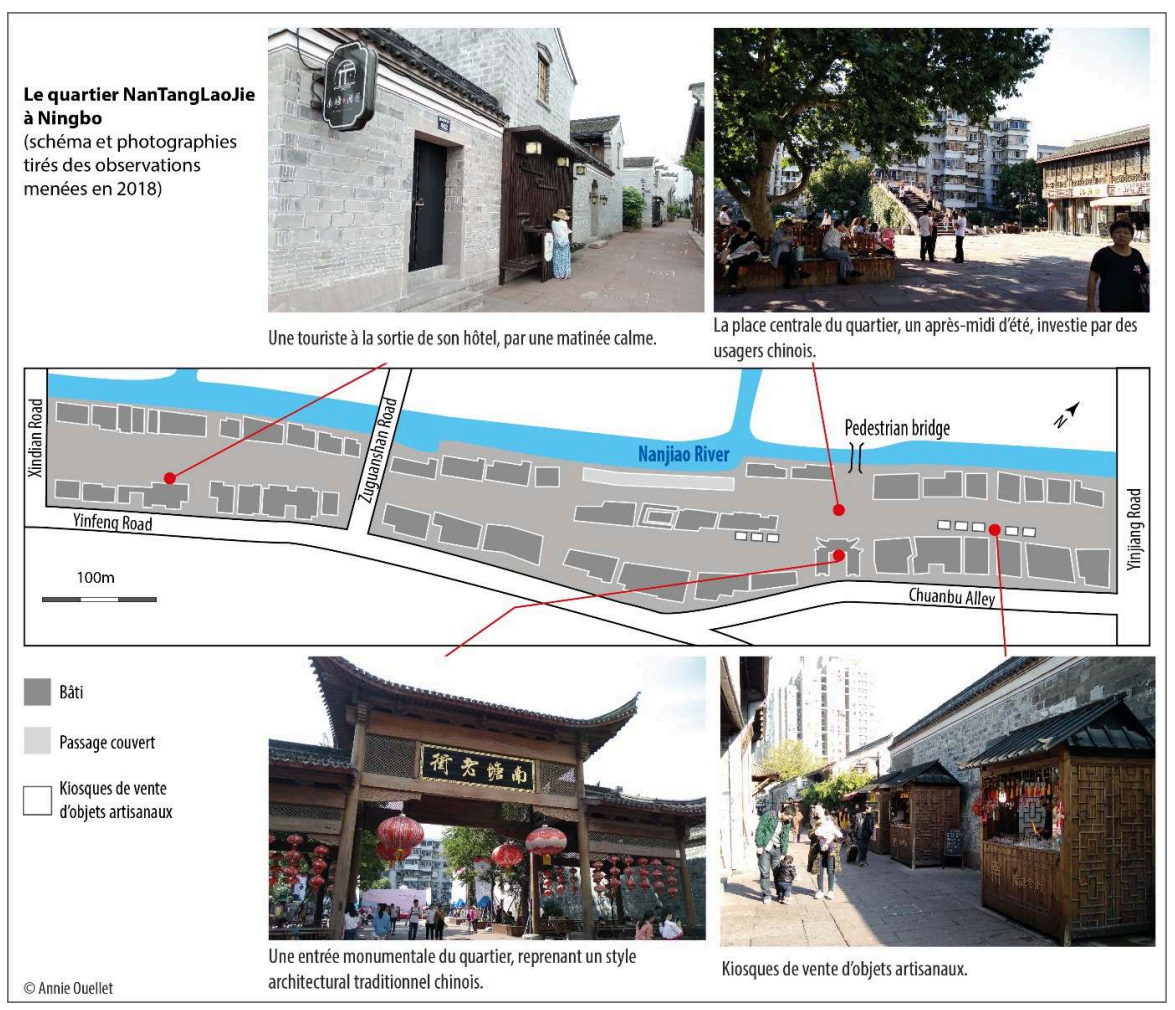

Sources : Basile Michel et Annie Ouellet, 2019. 
Figure 2. Terrain enquêté : M50 à Shanghai

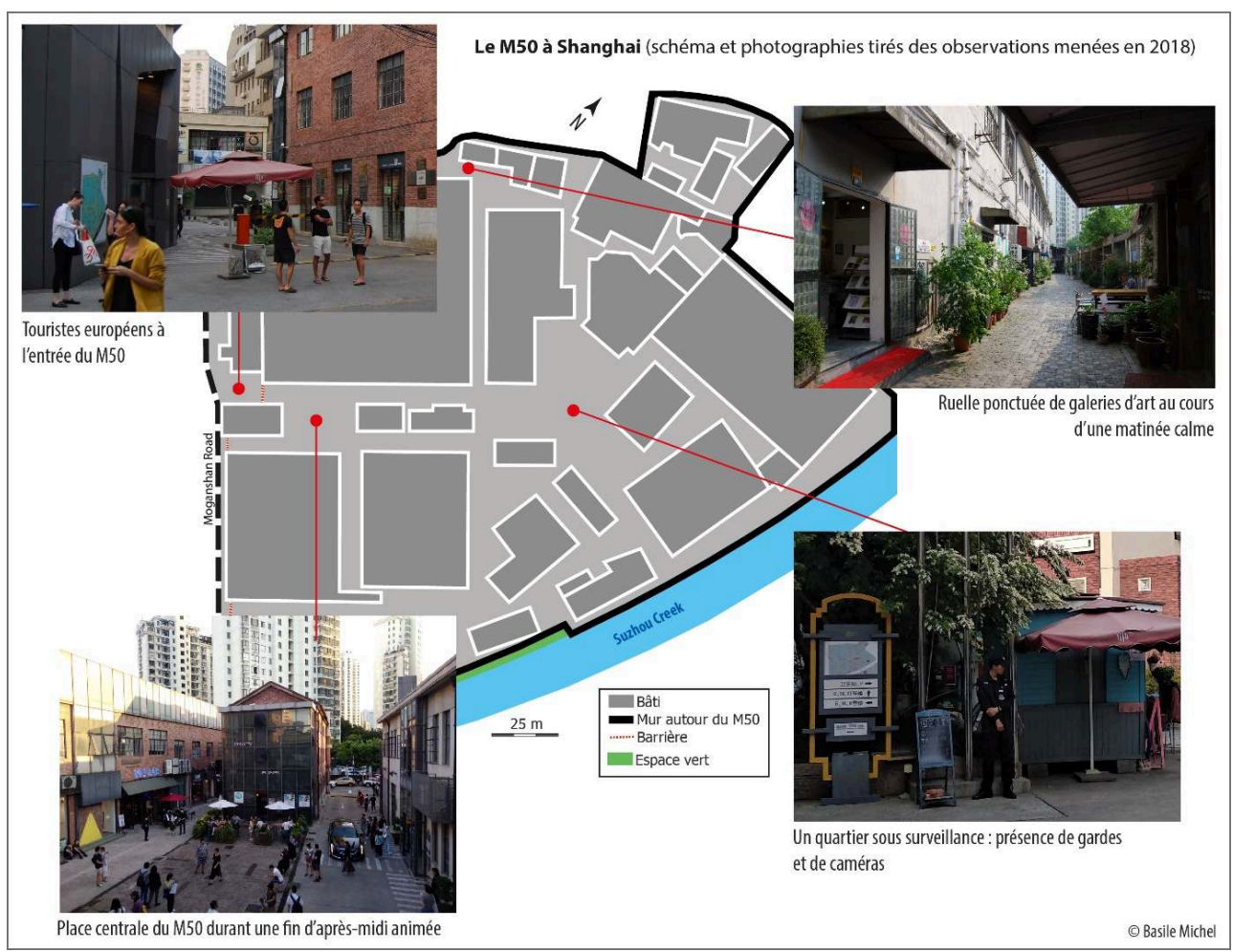

Sources : Basile Michel et Annie Ouellet, 2019.

Si l'observation autorise à saisir la réalité des pratiques, elle ne permet pas de comprendre le sens donné à celles-ci par les acteurs, ni les motivations qui en sont à l'origine (Petit, 2010), rendant ainsi nécessaire le croisement de ce premier outil avec un second : l'entretien.

\section{Les entretiens directifs et l'usage de l'anglais comme second contournement}

21 Face aux limites de l'observation, une seconde adaptation méthodologique a été réalisée dans le but de recueillir le discours des acteurs de NanTangLaojie et du M507. Cette adaptation repose sur l'utilisation, d'une part d'entretiens plus directifs et codifiés (dans les deux enquêtes), et d'autre part d'entretiens semi-directifs en anglais (au M50).

Dans le cas de NanTangLaojie, la quasi-totalité des visiteurs et commerçants étant chinois et ne maîtrisant que peu l'anglais, il a été nécessaire de faire appel à un tiers maîtrisant le mandarin et le français. Tel que mentionné précédemment, le travail de terrain a ainsi été mené en collaboration avec des étudiantes chinoises de Master $2^{8}$. Dans le contexte d'un terrain où la présence d'étrangers est peu commune et peut susciter l'étonnement voire la méfiance, cet accompagnement a pu constituer un élément facilitant la production du discours des personnes rencontrées.

Dans le but de contribuer à la fois à l'avancement du travail de terrain et à la formation à la recherche des étudiantes, le choix a été fait de mobiliser une enquête par entretiens directifs. Cet outil a été utilisé auprès des visiteurs et des commerçants du 
quartier. Il permet de faciliter le recueil de l'information auprès des acteurs en créant une trame précise de questions. Il simplifie aussi la traduction et la retranscription du matériau recueilli, les réponses étant plus courtes et plus factuelles que dans le cadre d'un entretien semi-directif. Malgré tout, des difficultés ont été rencontrées durant cette phase de traduction et de retranscription, principalement pour demeurer fidèle aux propos des personnes enquêtées.

Comme explicité précédemment, le terme patrimoine a connu de nombreuses évolutions tant en français qu'en chinois. Dans la grille d'entretien, nous avons fait le choix d'utiliser le terme chinois apparu le plus récemment, soit wenhua yichan (patrimoine culturel). Il fallait ensuite pouvoir traduire en français les propos des enquêtés. Au départ, chaque étudiante traduisait les propos recueillis une fois l'entretien terminé. Toutefois, il est apparu que, selon l'enquêtrice, un même mot employé en chinois par un enquêté pouvait être traduit différemment. Pour certaines questions, dont celles relatives à la description du quartier et à la conception du patrimoine, il a été nécessaire de revenir aux propos en mandarin des enquêtés, pour ensuite le traduire en français avec l'aide d'une seule personne, assurant ainsi la cohérence de l'ensemble du matériau discursif.

Dans le cas du M50, les phases d'observation ont permis de repérer la présence d'acteurs étrangers et chinois parlant anglais (des galeristes et des artistes). Ainsi, des entretiens semi-directifs en langue anglaise ont pu être réalisés, simplifiant la passation et le traitement de l'enquête. À la différence de NanTangLaoJie, les enquêtés au M50 n'éprouvaient pas d'étonnement face à la présence d'un étranger. Ce statut a même pu avoir un impact positif en levant tout soupçon d'accointance de l'enquêteur avec les autorité chinoises, permettant de libérer la parole des artistes et galeristes sur les questions politiques, certains n'hésitant pas à critiquer ouvertement les gestionnaires du M50 et les plans d'aménagement prévus par les pouvoirs locaux. Malgré ces aspects positifs, le recours à l'anglais n'est pas sans limite puisque l'usage d'une langue commune qui n'est la langue maternelle ni du chercheur ni de l'enquêté fait perdre en nuance et en précision. De plus, afin de ne pas exclure de l'analyse les acteurs parlant peu l'anglais, un procédé semblable à celui mis en place à NanTangLaoJie, à savoir l'utilisation d'entretiens directifs avec l'aide d'un tiers, a été mené9.

26 Ces contournements, que ce soit l'usage de l'anglais ou d'entretiens directifs, font perdre une partie de l'intérêt des entretiens semi-directifs, les réponses y étant moins libres et détaillées. Toutefois, ils facilitent l'entrée sur le terrain dans un contexte étranger et permettent de recueillir des données exploitables concernant les pratiques et les représentations des acteurs, et ce malgré les contraintes du terrain et nos propres limites en mandarin.

\section{Conclusion}

27 Cet article met en lumière un certain nombre de contraintes auxquelles, comme bien d'autres chercheurs en terrains étrangers, nous avons été confrontés lors de nos recherches en Chine. La non-maitrise de la langue locale, la courte durée des contrats de recherche et le faible soutien humain et financier sur place (absence d'interprètes professionnels par exemple) constituent autant de barrières entravant l'entrée sur le terrain et influençant directement la réalisation des recherches et les choix 
méthodologiques. Le contexte spécifique de la Chine joue également un rôle déterminant. Entre autres, le caractère autoritaire du régime en place incite le chercheur à la prudence voire à l'autocensure sur les questions politiques, les notions occidentales telles que le patrimoine et les clusters créatifs se doivent d'être réinterrogées au prisme des spécificités locales, et l'effet perturbateur de l'observateur est amplifié par la visibilité du chercheur étranger dans un contexte où la présence d'occidentaux est peu commune.

Face à ces contraintes et ces données contextuelles, des contournements méthodologiques ont été mis en place. L'observation a constitué une première entrée utile à la compréhension de l'organisation des espaces étudiés et des interactions sociales qui s'y déroulent. En complément, la réalisation d'entretiens en anglais ou d'entretiens directifs menés avec l'aide de personnes-ressources a permis de saisir le discours des acteurs. S'ils n'effacent pas complètement la frustration induite par la barrière de la langue et les contraintes temporelles, humaines et financières, ces contournements permettent malgré tout une première approche du terrain en contexte étranger. La comparaison entre les deux recherches mobilisées dans cet article permet, d'une part, de renforcer la portée des constats sur les difficultés à mener des recherches en terrain étranger dans des conditions contraignantes et, d'autre part, de montrer une partie de la diversité des arrangements et bricolages qui peuvent être mis en place pour faire face à ces difficultés.

\section{BIBLIOGRAPHIE}

ARBORIO A-M. (2007), « L'observation directe en sociologie : quelques réflexions méthodologiques à propos de travaux de recherches sur le terrain hospitalier », Recherche en soins infirmiers, vol. 3, n 90 , pp. 26-34.

ARBORIO A-M., FOURNIER P. (2015), L'observation directe, Paris, Armand Colin.

CABESTAN J-P. (2014), Le système politique chinois. Un nouvel équilibre autoritaire, Paris, Presses de Sciences Po.

CALBÉRAC Y. (2010), Terrains de géographes. Géographes de terrain. Communauté et imaginaire disciplinaires au miroir des pratiques de terrain des géographes français du XXe siècle, Thèse de doctorat en géographie, Université Lumière Lyon II.

CLAVAL P. (2013), « Le rôle du terrain en géographie. Des épistémologies de la curiosité à celles du désir ", Confins, $n^{\circ} 17$, https://journals.openedition.org/confins/8373, consulté le 2 septembre 2018.

COËFFE V., MORICE J.-R. (2017), « Patrimoine sans limite ? La mondialisation du tourisme comme opérateur d'un « tout-patrimoine » », L’Information géographique, vol. 81, n², pp. 32-54.

ESO (collectif) (2008), « L'espace social : méthodes et outils, objets et éthique(s) », ESO Travaux \& Documents, $\mathrm{n}^{\circ} 27$. 
KEANE M. (2011), China's New Creative Clusters: Governance, Human Capital and Investment, Londres, Routledge.

KEANE M. (éd.) (2016), Handbook of Cultural and Creative Industries in China, Cheltenham et Northampton : Edward Elgar.

LAZZAROTTI O. (2003), « Patrimoine », in Lévy J., Lussault M. (dir.), Dictionnaire de la géographie et de l'espace des sociétés, Paris, Belin, pp. 692-693.

LEMERCIER C., OLLIVIER C., ZALC C. (2013), « Articuler les approches qualitatives et quantitatives. Plaidoyer pour un bricolage raisonné » in Hunsmann M., Kapp S. (dir.), Devenir chercheur. Écrire une thèse en sciences sociales, Paris, Éditions EHESS, pp. 125-143.

MICHEL B. (2017), Les quartiers créatifs : une dynamique de club. Analyse croisée des quartiers des Olivettes (Nantes), du Panier (Marseille) et Berriat (Grenoble), Thèse de doctorat en géographie, Université d'Angers.

MICHEL B. (2018), « Les quartiers créatifs : construction de clubs de travailleurs créatifs. Analyse croisée des quartiers du Panier (Marseille) et des Olivettes (Nantes) ", Annales de Géographie, $n^{\circ} 721$, pp. 227-253.

MORANGE M., SCHMOLL C., TOUREILLE E. (2016), Les outils qualitatifs en géographie. Méthodes et applications, Paris, Armand Colin.

OUELLET A. (2015), « Dinan en représentation(s). Patrimonialisation et mise en tourisme d'une petite ville historique de Bretagne », Norois, $\mathrm{n}^{\circ} 234$, pp. 47-63.

OUELLET A. (2017), Coprésence et rapports à l'espace dans les petites villes touristiques et patrimoniales. Lecture croisée de Dinan (Côtes-d'Armor) et Sarlat-la-Canéda (Dordogne), Thèse de doctorat en géographie, Université d'Angers.

PETIT E. (2010), « Du fil de l'eau en fils à retordre. Comment bricoler des techniques de terrain protéiformes en une méthodologie qualitative cohérente en géographie ? », L'Information géographique, vol. 74, n¹, pp. 9-26.

RODDAZ J.-M. (2017), « Le financement de la recherche sur projets : pourquoi et comment ? », Mélanges de la Casa de Velázquez, vol. 47, n¹, pp. 321-328.

SANJUAN T. (2008), « Les approches du fait chinois par la géographie française », in Sanjuan T. (dir.), Carnets de terrain. Pratique géographique et aires culturelles, Paris, L'Harmattan, pp. 95-116.

VIVET J., GINISTY K. (2008), « Les biais, terrain de savoirs ? Expériences africaines », A travers l'espace de la méthode : les dimensions du terrain en géographie, Juin 2008, Arras, France, https://halshs.archives-ouvertes.fr/halshs-00408163/document, consulté le 28 avril 2019.

ZHANG C. (2017), « L'évolution historique de la notion de "patrimoine" en Chine », L'Information géographique, vol. 81, n², pp. 75-93.

ZHENG J., CHAN R. (2014), « The Impact of "Creative Industry Clusters" on Cultural and Creative Industry Development in Shanghai », City, Culture and Society, n5, pp. 9-22.

\section{NOTES}

1. L'article a été rédigé sans écriture inclusive afin d'éviter d'alourdir le texte à l'exception des mentions directes aux deux auteur-e-s.

2. Parmi ces écrits : les textes tirés du colloque « À travers l'espace de la méthode : les dimensions du terrain en géographie » qui s'est tenu à Arras en 2008, le numéro thématique du Bulletin de 
l'Association des Géographes Français («Le «terrain » pour les géographes », 2007, vol.84, n4) et celui de l'Information Géographique («Le terrain», 2010, vol.74, n¹), ainsi que bon nombre d'articles parus dans Carnets de géographes.

3. Les auteur-e-s ont été recruté-e-s en tant qu'enseignant-e - chercheur-e pour une durée inférieure à une année au sein de l'Institut conjoint des universités de Ningbo et d'Angers à Ningbo (Zheijiang, Chine).

4. Une note de l'Ambassade de France en Chine détaille les conditions de travail des enseignantschercheurs des universités chinoises, mettant notamment en avant une forte pression à la publication: $\quad$ https://www.galaxie.enseignementsup-recherche.gouv.fr/ensup/pdf/ EC_pays_etrangers/Asie/Chine.pdf, consulté le 11 avril 2019.

5. L'emploi des termes «étranger» ou "occidental» exige prudence et précaution car ils engagent un découpage du monde dont les limites sont à la fois critiquables et difficilement identifiables. La diversité des populations regroupées sous ce vocable est grande, tout comme celle des "chinois". Nous précisons donc que les termes "occidentaux ", "étrangers » et « chinois » sont utilisés dans cet article afin de différencier de manière sommaire les touristes chinois des touristes étrangers, très majoritairement occidentaux sur nos terrains d'étude (identifiables par leur apparence physique et les langues employées). Cette généralisation se justifie ici au vu de l'un des objectifs de nos recherches, à savoir caractériser les formes de tourisme qui se développent au M50 et à NanTangLaoJie, et notamment le degré d'internationalisation de ces lieux touristiques en évaluant leur fréquentation par des touristes internationaux en plus des touristes chinois.

6. Au-delà de l'observation, les entretiens ont permis d'identifier les résidents, les touristes et autres types d'usagers des quartiers.

7. En complément des observations et des entretiens, une analyse d'articles de presse et de documents d'urbanisme a été réalisée.

8. Nous remercions Liang Min, Liu Jingru, Xirou Yiang et Xu Linying pour leur travail.

9. Nous remercions Shi Yuanyuan pour son travail.

\section{RÉSUMÉS}

La pratique de terrain, centrale dans le travail de recherche en géographie, peut s'avérer plus ou moins complexe et contraignante. Elle est en effet liée à de multiples facteurs, tels l'accès aux acteurs, la disponibilité des données, la connaissance de la langue et des codes locaux par le chercheur, etc. Cet article vise à mettre en lumière à la fois les contraintes liées à la pratique du terrain en contexte étranger, et les moyens, les arrangements et les bricolages qui peuvent être mis en place pour les surmonter. Pour cela, deux recherches menées en Chine par des chercheure-s nord-américaine et français sont mobilisées. L'une porte sur la patrimonialisation et la mise en tourisme d'un quartier de la ville de Ningbo, l'autre sur les dynamiques urbaines, artistiques et touristiques du quartier créatif M50 de Shanghai. Dans les deux cas, c'est la non-maîtrise du mandarin qui a constitué la principale contrainte, bien que d'autres se soient également ajoutées (contrats courts, contexte politique autoritaire, etc.). Le croisement de ces deux exemples, où les contraintes initiales et les éléments de contextes sont similaires, permet de mettre en évidence la diversité des contournements mis en place en fonction de la spécificité des terrains appréhendés. 
Fieldwork practice, central to geography research, can be more or less complex and constraining. It is indeed linked to multiple factors, such as access to stakeholders, availability of data, knowledge of the language and local codes by the researcher, etc. This article aims to highlight both the constraints of fieldwork practice in foreign context, and the measures, arrangements and methodological "deals" that can be put in place to overcome these constraints. For that, two researches carried out in China by researchers from North America and France are mobilized. The first one focuses on heritage-making and tourism development in an urban neighborhood in the city of Ningbo. The other one explores the urban, artistic and tourist dynamics of Shanghai's creative district M50. In both cases, it was the lack of Mandarin-language skills that was the main constraint, although others were also added (short-term contracts, authoritarian political context, etc.). The crossing of these two examples, where the initial constraints and contextual elements are similar, highlights the variation of workarounds implemented depending on the specificity of fieldworks.

\section{INDEX}

Thèmes : Carnets de terrain

Mots-clés : contraintes, contournements méthodologiques, terrain étranger, Chine

Keywords : Constraints, Methodological workarounds, Fieldwork in foreign countries, China

\section{AUTEURS}

\section{BASILE MICHEL}

Laboratoire ESO (UMR CNRS 6590), Institut conjoint des universités de Ningbo et d'Angers, basile.michel@gmail.com

\section{ANNIE OUELLET}

Laboratoire ESO (UMR CNRS 6590), Institut conjoint des universités de Ningbo et d'Angers, annieouellet22@gmail.com 